\title{
Feature-negative effect in rats' discrimination learning in the runway
}

\author{
STEVEN J. HAGGBLOOM \\ Arkansas State University, State University, Arkansas
}

\begin{abstract}
In two experiments, rats were trained on a successive go/no-go discrimination problem in the runway in which the positive (S+) and negative (S-) discriminanda were differentiated by the presence or absence of a distinctive feature. The feature in Experiment 1 was a series of flashing lights over the runway. In Experiment 2, the feature was a pretrial reinforcement (Phase 1), or pretrial reinforcement versus pretrial nonreinforcement (Phase 2). The feature signaled $\mathbf{S}+$ trials in feature-positive (FP) groups and S- trials in feature-negative (FN) groups. The original discrimination was reversed in Phase 2 of both experiments. With the exception of the pretrial nonreinforcement groups in Experiment 2, there was an asymmetry in discrimination learning in both phases of both experiments favoring superior discrimination learning by FN subjects over FP subjects, a feature-negative effect. Implications of the results for an information processing account of asymmetries in learning feature discriminations are discussed.
\end{abstract}

Jenkins and Sainsbury (1969) reported an asymmetry in pigeons' learning of a successive go/no-go discrimination in which the positive $(\mathrm{S}+)$ and negative $(S-)$ discriminanda were differentiated by a distinctive stimulus (feature), presented on $\mathrm{S}+$ trials in the feature-positive (FP) case and on $S$ - trials in the feature-negative (FN) case, in the direction of faster learning of FP discriminations than FN discriminations. This asymmetry, called the featurepositive effect (FPE), is quite pervasive, having been obtained across a considerable range of experimental procedures, learning tasks, types of features, and species (see Hearst, 1978, in press, for reviews). The generality of the FPE has been taken to indicate that a fundamental psychological principle (or bias), perhaps due to the innate organization of the nervous system, underlies its occurrence (Hearst, 1978; Newman, Wolff, \& Hearst, 1980). Hearst (in press) has suggested that the FPE may be related to some effects in human learning and information processing characterized by a bias favoring the presence over the absence of a stimulus as a source of information.

The two experiments reported here investigated feature-discrimination learning in rats, employing for the first time a successive go/no-go discrimination problem in the runway. Previous feature-learning experiments with rats have demonstrated a FPE in appetitive barpress situations (Crowell \& Bernhardt, 1979; Halgren, 1974, Lea, 1974) and in conditioned suppression (Reberg \& Leclerc, 1977). Whether the

These experiments were supported by a Faculty Research Grant from Arkansas State University. Requests for reprints should be addressed to the author, Department of Psychology, Arkansas State University, P.O. Box 2127, State University, Arkansas 72467.
FPE will occur in the runway is relevant to conclusions about the generality of the FPE across discrimination tasks as well as to theoretical explanations of the FPE.

\section{EXPERIMENT 1}

Rats were trained on either an FP or FN discrimination in Phase 1 and were then shifted to the opposite (reversed) discrimination in Phase 2. The feature was a series of flashing lights suspended above the length of the runway. The feature was presented on $\mathrm{S}+$ trials in Group FP and on S- trials in Group FN. A control group, Group C, experienced the feature on rewarded trials on odd-numbered days of training and on nonrewarded trials on even-numbered days of training.

\section{Method}

Subjects. The subjects were 30 male rats, 90 days old at the beginning of the experiment, bred in the laboratory from Holtzman stock.

Apparatus. The apparatus consisted of a straight black runway, $130 \mathrm{~cm}$ long $\times 9 \mathrm{~cm}$ high and wide. The first $25 \mathrm{~cm}$ made up the startbox, and the last $25 \mathrm{~cm}$ constituted the goalbox. The startbox was separated from the runway by a manually operated guillotine door. The goalbox, separated from the runway in the same manner, contained an unpainted wooden goal cup. Start times were recorded from the opening of the startbox door, which triggered a $.01-\mathrm{sec}$ clock, to a point $30 \mathrm{~cm}$ into the runway. Run and goal times were measured over the next 40 and $30 \mathrm{~cm}$, respectively. The offset of the first clock and the operation of the run and goal clocks were controlled by photoelectric circuitry.

A series of six $24-\mathrm{V}$ dc lights was suspended $2 \mathrm{~cm}$ above the hardware-cloth lid of the runway. The lights were located at $24.5-\mathrm{cm}$ intervals, beginning $2 \mathrm{~cm}$ past the startbox door and terminating directly over the goal cup $2 \mathrm{~cm}$ in front of the end wall of the goalbox. The lights were controlled by an interval timer activated by 
the opening of the startbox door (on feature trials) and set so that the lights would flash repeatedly for $.5 \mathrm{sec}$ on $/ .5 \mathrm{sec}$ off.

Procedure. The rats were housed individually throughout the experiment. All food was removed from each rat's cage 14 days prior to the beginning of experimental training. Thereafter, each rat received $12 \mathrm{~g}$ of lab chow per day minus the amount of food received in the runway. Water was always available. On Days 12-14 of deprivation, the rats were handled in squads of three for $3 \mathrm{~min}$ per squad and subsequently fed $1045-\mathrm{mg}$ Noyes food pellets from a small dish placed in the home cage. Ten rats were assigned randomly to each of three groups, and discrimination training began on Day 15 of deprivation.

Each rat received three $S+$ and three $S-$ trials on each of 18 days of original discrimination learning (Phase 1) and 17 days of reversal learning (Phase 2 ). ${ }^{1}$ The following six irregular sequences of $S+$ and $S-$ trials were used recursively over successive 6-day blocks of training: $S+S+S-S-S+S-, S-S+S-S-S+S+$, $\mathrm{S}+\mathrm{S}-\mathrm{S}+\mathrm{S}-\mathrm{S}+\mathrm{S}-, \mathrm{S}-\mathrm{S}-\mathrm{S}+\mathrm{S}+\mathrm{S}-\mathrm{S}+, \mathrm{S}+\mathrm{S}-\mathrm{S}+\mathrm{S}+\mathrm{S}-\mathrm{S}-$ $S-S+S-S+S-S+$. Reinforcement on $S+$ trials consisted of 10 $45-\mathrm{mg}$ Noyes pellets. On $S-$ trials, the subjects were confined to the unbaited goalbox for $20 \mathrm{sec}$.

The feature (flashing light), the only stimulus that reliably predicted reinforcement, was presented on $S+$ trials to Group FP and on $\mathrm{S}$ - trials to Group FN in Phase 1. Group C experienced the feature on rewarded $(S+)$ trials on odd-numbered days of training and on nonrewarded (S-) trials on even-numbered days of training. Thus, the feature did not predict reinforcement for Group C. In Phase 2, the feature was presented on S- trials to Group FP (now designated Group FP $\rightarrow$ FN) and on $S+$ trials to Group FN (now designated Group FN $\rightarrow$ FP). Group $C$ was not run in Phase 2.

The subjects were given their daily trials in squads consisting of three rats from each group in Phase 1 and of two rats from each group in Phase 2. The order in which the subjects were run within squads was randomized daily. The order of successive squads was held constant across days. Within a squad, each rat received Trial 1 before any rat received Trial 2 , and so on. This procedure resulted in an intertrial interval of $2-3 \mathrm{~min}$.

To initiate a trial, the startbox door was opened approximately 3 sec after the rat was placed in the startbox, regardless of the rat's orientation. A maximum time of $\mathbf{3 0} \mathrm{sec}$ was allowed in each section of the alley. If $30 \mathrm{sec}$ was exceeded in any alley section, the additional time was added to the time score of the next section forward. If the animal did not enter the goalbox within $90 \mathrm{sec}$, it was placed in the goalbox.

\section{Results and Discussion}

The times for each subject on each trial were converted to speeds in $\mathrm{cm} / \mathrm{sec}$. Since the results were essentially the same in each alley section, only analyses based on speeds over the total alley are presented here. Figure 1 shows the mean difference between speeds in $\mathrm{S}+$ and $\mathrm{S}-$ trials in blocks of 3 days for Groups FP and FN in Phase 1 (left panel) and for those same groups shifted to the reversed discrimination and now designated FP $\rightarrow$ FN and FN $\rightarrow$ FP in Phase 2 (right panel). The left panel of Figure 1 also shows the mean difference between speeds on feature-present versus feature-absent trials for Group C.

As can be seen in Figure 1, both the original discrimination and the reversal were learned more rapidly in the FN condition than in the FP condition. An analysis of variance (ANOVA), with groups (2) as a between-subjects variable and days (18) as a withinsubjects variable applied to the difference scores for

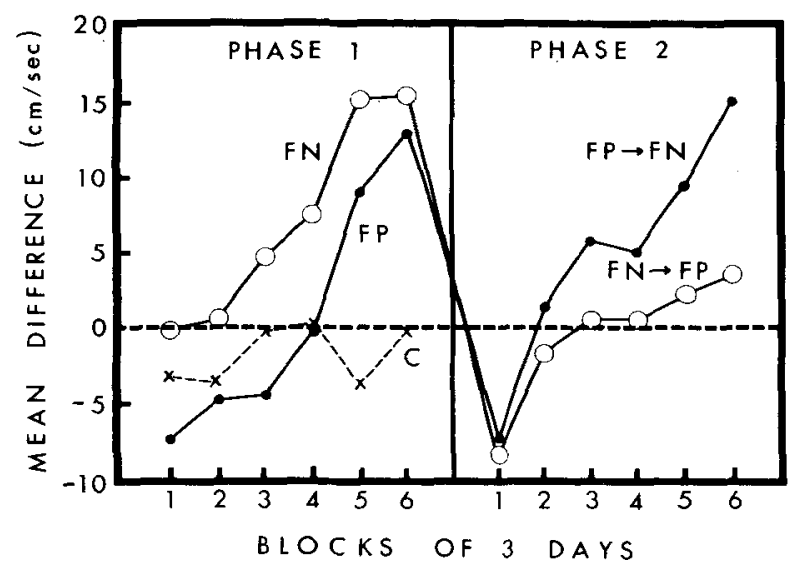

Figure 1. Mean difference between speeds in $S+$ and $S-$ for Groups FP, FN, and $C$ in Phase 1 (left panel) and for Groups FP $\rightarrow$ FN and FN $\rightarrow$ FP in Phase 2 (right panel). Block 6 in Phase 2 represents a mean over only 2 days.

Groups FP and FN in Phase 1 yielded reliable effects for groups $[F(1,18)=4.73, p<.05]$ and days $[F(17,306)$ $=7.74, \mathrm{p}<.001]$, but no groups $\times$ days interaction $(F<1)$. Separate ANOVAs involving all three groups and subsequent simple effects of discriminanda applied to running speeds in $\mathrm{S}+$ and $\mathrm{S}-$ on each of the six trial blocks shown in the left panel of Figure 1 showed that $S+$ speeds were reliably faster than $S-$ speeds in Group FN by Block $3[F(1,27)=$ $6.49, \mathrm{p}<.05$ ], but not until Block 5 in Group FP $[F(1,27)=5.30, p<.05]$. There was a slight tendency for Group $\mathrm{C}$ to run more slowly on feature trials than on nonfeature trials, but that difference was reliable only on Block $2[\mathrm{~F}(1,27)=5.65, \mathrm{p}<.05]$. These analyses support the observation that the discrimination was acquired more readily in Group FN than in Group FP in Phase 1.

A groups (2) $\times$ day (11) ANOVA applied to difference scores over the last four blocks (three blocks of 3 days and one of 2 days) of Phase 2 showed that the difference in level of performance achieved on the reversed discrimination problem between Groups $\mathrm{FP} \rightarrow \mathrm{FN}$ and $\mathrm{FN} \rightarrow \mathrm{FP}$ was reliable $[\mathrm{F}(1,18)=4.89$, $\mathrm{p}<.05]$. The days effect was also reliable $[\mathrm{F}(10,180)$ $=2.78, p<.011$, but the groups $\times$ days interaction was not $(F<1)$. Additional ANOVAs and subsequent simple effects of discriminanda for both groups showed that Group FP $\rightarrow$ FN ran reliably faster in $S+$ than in $S-$ by Block $3[F(1,18)=5.60$, p < .05]. Group FN $\rightarrow$ FP, on the other hand, did not run reliably faster in $S+$ than in $S-$ on any trial block [largest $F(1,18)=1.12$ for Block 6 ]. These analyses support the observation that the reversed discrimination, like the original discrimination, was acquired more readily in the FN condition than in the FP condition.

Of some concern here is the possibility that discrimination learning was facilitated in the FN condition and impaired in the FP condition because the 
feature may have been aversive and its presentation could have caused a reduction in $S+$ speeds in Group FP and a reduction in S- speeds in Group FN. Indeed, Group FP ran slightly more slowly than Group FN in S+ over much of Phase 1. However, Group FP also ran more slowly than Group FN in S- (hence, the presentation of difference scores here). There are several reasons for discounting the possibility that the superior discrimination learning obtained here in the FN condition, a feature negative effect (FNE), was due to the aversiveness of the feature. First, Group C showed only a slight and inconsistent tendency to run more slowly on feature than on nonfeature trials. Second, in Phase 2 Group $\mathrm{FN} \rightarrow \mathrm{FP}$ continued to run faster in $\mathrm{S}+$ than Group $F P \rightarrow F N$, even though it was now Group FN $\rightarrow$ FP that experienced the feature on $S+$ trials. Finally, while collecting the data for this experiment, I did not notice any behaviors that would suggest that the light had aversive properties.

\section{EXPERIMENT 2}

Bottjer and Hearst (1979) obtained, with pigeons, a FPE in an autoshaping paradigm in which the presence versus the absence of a pretrial food delivery served as the feature. Since a pretrial food delivery has the distinct advantage, in view of the preceding discussion, of not being aversive, it was employed as the feature in Phase 1 of Experiment 2. Immediately preceding feature trials, the subjects were placed in a box, located next to the runway, that was baited with the same number of reinforcement pellets employed as a reinforcement on $\mathrm{S}+$ trials. In Phase 2, the discrimination was reversed, as in Experiment 1 . Half of the subjects shifted from a FP to a FN discrimination, and half of those shifted from a FN to a FP discrimination continued to receive a reinforced placement as the feature. The feature for the remainder of the subjects in both groups in Phase 2 was a nonreinforced placement.

\section{Method}

Subjects. The subjects were 20 male rats of the same description as those in Experiment 1.

Apparntus. The apparatus was the same as in Experiment 1 except that the lights were removed from over the runway and a $20 \times 25 \mathrm{~cm}$ plywood box with $20-\mathrm{cm}$-high sides was located adjacent to the side of the runway.

Procedure. Except as noted, all procedures prior to the beginning of discrimination training were the same as in Experiment 1. The rats were handled in squads of four for $4 \mathrm{~min}$ per squad and were fed five 45-mg Noyes pellets in their home cages on Days 1214 of deprivation. The rats were divided randomly into two equal groups, and discrimination training began on Day 15 of deprivation.

Each rat received 10 days of discrimination training in Phase 1 and 12 days of reversal training in Phase 2 with the same sequences of $\mathbf{S}+$ and $\mathbf{S}$ - trials used in Experiment 1 . In Phase 1, rats in Group FP were placed in the plywood box, which was baited with five $45-\mathrm{mg}$ food pellets placed in a baby-food-jar lid, immediately preceding $\mathbf{S}+$ trials. After consuming the pretrial reinforcement, the subject was removed from the box and placed in the startbox of the runway. Rats in Group FN experienced the same procedure preceding $S-$ trials.

In Phase 2, the subjects given FP training in Phase 1 were shifted to the FN condition and those given FN training were shifted to the FP condition. One-half of the subjects in each reversal condition continued to receive a pretrial reinforcement as the feature [Groups $\mathbf{F P} \rightarrow \mathrm{FN}(\mathrm{R})$ and $\mathrm{FN} \rightarrow \mathrm{FP}(\mathrm{R})$ ]. The remaining subjects received a pretrial nonreinforcement as the feature. That is, the plywood box was empty [Groups FP $\rightarrow$ FN(N) and FN $\rightarrow \mathrm{FP}(\mathrm{N})]$.

Trials were administered to subjects in squads of four, with each containing one subject from each of the Phase 2 conditions. The intertrial interval was 3-4 min. All other procedures were identical to those employed in Experiment 1.

\section{Results and Discussion}

All time scores were converted to speeds in $\mathrm{cm} / \mathrm{sec}$. The results were the same in each alley section, so only total speeds are reported here. Figure 2 shows the mean difference between speeds in $S+$ and $S-$ for both Phase 1 conditions in the left panel and for all four Phase 2 conditions in the right panel.

Looking at the left panel of Figure 2, it can be seen that, as in Experiment 1, the original discrimination was acquired more rapidly in the FN condition than in the FP condition. A groups (2) $\times$ days (10) ANOVA applied to difference scores in Phase 1 yielded significant effects for groups [F $(1,18)=$ $14.24, \mathrm{p}<.01]$ and days $[\mathrm{F}(9,162)=13.71, \mathrm{p}<.01]$, but not for groups $\times$ days $[F(9,162)=1.68]$. Separate ANOVAs and subsequent simple effects of discriminanda at each group on each of the five trial blocks shown in Figure 2 for Phase 1 showed that Group FN ran reliably faster in $S+$ than in $S-$ by Block 2 $[F(1,18)=12.32, p<.01]$, but that Group FP did not run faster in $S+$ than in $S-$ until Block $5[F(1,18)$ $=8.21, \mathrm{p}<.05$ ].

Looking now at the right panel of Figure 2, it can be seen that discrimination learning in groups given

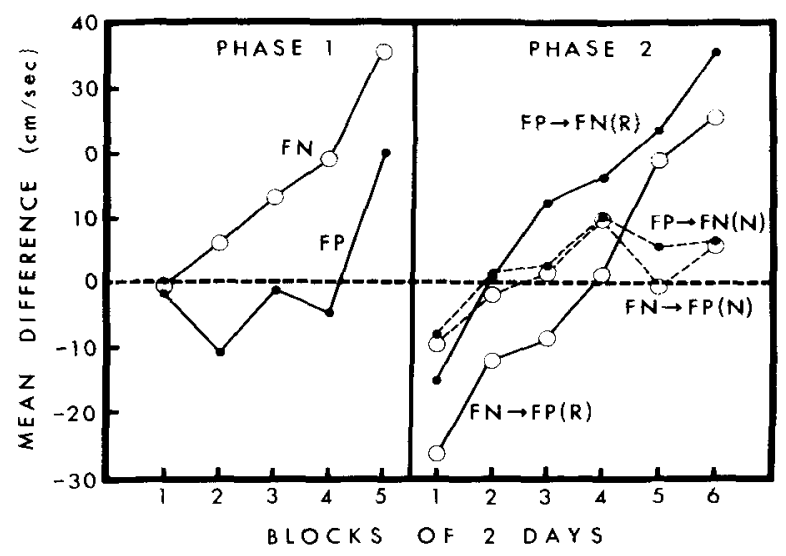

Figure 2. Mean difference between speeds in $\mathbf{S}+$ and $\mathbf{S}-$ for Groups FP and FN in Phase 1 (left panel) and for Groups FP $\rightarrow$ FN(R), FN $\rightarrow$ FP(R), FP $\rightarrow$ FN(N), and FN $\rightarrow$ FP(N) in Phase 2 (right panel). 
pretrial reinforcements as the feature was better in the FN condition [Group FP $\rightarrow$ FN(R)] than in the FP condition [Group FN $\rightarrow$ FP(R)]; but, in groups given pretrial nonreinforcements as the feature, discrimination learning was inferior to that in both pretrial reinforcement conditions, and there was essentially no difference between the FN and FP conditions.

An ANOVA with FP vs. FN (2) and type of feature (2) as between-subjects factors and days (12) as a within-subjects factor was applied to the difference scores in Phase 2. Consistent with what can be seen in Figure 2, there was a reliable interaction between the FP vs. FN treatment and type of feature $[F(1,16)=$ $7.74, p<.05]$. Simple effects showed that FN discrimination learning was superior to FP learning in groups given pretrial reinforcements as the feature $[F(1,16)=21.47, p<.01]$, but not in groups given pretrial nonreinforcements as the feature $(F<1)$. Simple effects also showed that Group FP $\rightarrow$ FN(R) performed better than Group $\mathrm{FP} \rightarrow \mathrm{FN}(\mathrm{N})[\mathrm{F}(1,16)=$ $9.50, \mathrm{p}<.01]$. Although the simple effect difference between Groups $F N \rightarrow F P(R)$ and $F N \rightarrow F P(N)$ was not reliable $(F<1)$, the fact that discrimination learning was eventually better in both groups given pretrial reinforcements than in the groups not given pretrial reinforcement was reflected in a reliable interaction between type of feature and days $[F(11,176)=8.16$, $\mathrm{p}<.011$.

Separate ANOVAs and subsequent simple effects of discriminanda at each group showed that Group FP $\rightarrow$ FN(R) ran reliably faster in $S+$ than in $S-$ by Block $3[F(1,16)=15.36, p<.01]$, but that Group $F N$ $\rightarrow F P(R)$ did not run faster in $\mathbf{S}+$ than in $S-$ until Block $5[\mathrm{~F}(1,16)=12.60, \mathrm{p}<.01]$. Groups $\mathrm{FP} \rightarrow$ FN(N) and $F N \rightarrow F P(N)$ ran faster in $S+$ than in $S-$ only on Block 4 [Fs $(1,16)=27.22$ and 29.0 , ps $<.01$, respectively].

\section{GENERAL DISCUSSION}

In two experiments, in which rats learned a successive go/no-go discrimination in the runway, FN subjects learned to discriminate substantially better than FP subjects-a FNE. The FNE occurred in both the original discrimination learning phase and a reversal learning phase in both experiments. As indicated above, FP superiority is the usual finding in feature learning experiments. There have been occasional failures prior to this to obtain a FPE (e.g., Farthing, 1971; Sainsbury, 1973), but only one previously reported instance of FN superiority (Hearst, 1969), and that finding was thought by Hearst not to represent a genuine FNE, but to have been a consequence of the type of preliminary exposure to the discriminanda his birds received.

The reversal learning results obtained here are consistent with those of feature-learning experiments re- porting a FPE in Phase 1 and then employing a reversal phase in that the discrimination acquired most readily in the reversal phase was the same discrimination acquired most readily in original learning (see, e.g., Hearst, 1978).

The FNEs obtained here cannot readily be explained in terms of procedural differences between the present experiments and experiments reporting FPEs. For example, most feature-learning experiments have employed a preliminary training phase in which subjects received nondifferential reinforcement for responding to one or both discriminanda or some component of the subsequent discriminative stimuli, say, the feature or the common element. In contrast, there was no such preliminary training phase in the present experiments. An examination of the literature since 1969, though, shows that the FPE occurs under a wide range of preliminary training conditions, including no pretraining (Bottjer \& Hearst, 1979, Experiment 2; Pace \& McCoy, 1981).

Similarly, the FNE obtained here cannot be attributed simply to the nature of the stimuli used as features. Jenkins and Sainsbury (1969) employed discrete visual stimuli as features and common stimulus elements, for example, a display containing three dots versus one containing two dots and a star. Many subsequent experiments followed suit and employed discrete, localized visual cues as the feature and common elements. The flashing light employed as a feature in Experiment 1 here is probably best viewed as a diffuse, rather than a localized, visual cue. Halgren (1974), however, obtained a FPE with a diffuse (auditory) stimulus as the feature. And, except for differences in the method of providing a pretrial food delivery, the feature in Experiment 2 here was essentially that used by Bottjer and Hearst (1979). That procedure produced FN superiority in the present case and FP superiority in Bottjer and Hearst's experiments.

Virtually all feature-learning experiments have employed some discrete stimulus or set of stimuli as the common element(s). In contrast, the common elements in the present experiments consisted of contextual cues, for example, the runway and background sources of stimulation. However, there appears to be no empirical or theoretical basis for rejecting contextual cues as viable common stimulus elements in feature-learning discriminations. Indeed, contextual cues exercise substantial control over animal behavior (see Riccio, Urda, \& Thomas, 1966) and are often treated in the same way as discrete stimulus events (Hinson, 1982). Moreover, Terry and Wagner (1975) apparently obtained a FPE in an aversive classical conditioning paradigm employing a pretrial reinforcement as the feature and no discrete stimulus as the common element.

In most feature-learning experiments, subjects must physically contact, that is, peck at, either the 
feature or one of the common stimulus elements to complete the response requirement. This allows subjects to direct their behavior and/or attention toward the feature on $\mathrm{S}+$ trials in the FP condition and away from the feature on $S-$ trials in the FN condition. Such directed behavior, called sign-tracking, often accompanies feature discrimination learning (Hearst, 1978; Hearst \& Jenkins, 1974). In the present experiments, subjects could not direct their behavior toward or away from the flashing light and pretrial reinforcement employed as features, and so signtracking was not possible. In contrast to the FNE obtained here, however, others have obtained a FPE under conditions that precluded sign-tracking (Bottjer \& Hearst, 1979; Halgren, 1974; Lea, 1974).

As indicated by the preceding discussion, the procedures employed in the present experiments differed in many respects from those traditionally used in feature-learning investigations. Except for the use of a runway, however, those procedures (diffuse feature, pretrial reinforcement as a feature, no preliminary training, conditions that preclude signtracking) have all been employed, either alone or in various combinations, in investigations reporting a FPE. Evidently, then, the FNE obtained here represents a genuine instance of FN superiority.

Why FN learning might be superior to FP learning in one situation and FP learning would be superior in another is not clear. Perhaps a better understanding of the effects on feature learning of some of the variables discussed above will shed light on this question. A more fundamental question is whether the occurrence of a FNE is necessarily problematic for current theoretical explanations of feature learning which, of course, have been developed to explain the FPE. The view advocated here is that the FNE cannot only be accommodated by one popular sort of explanation of feature learning, one that attributes the FPE to an information-processing bias (Hearst, 1978, in press); in addition, on those same theoretical grounds, we should expect to identify situations in which FN learning is superior to FP learning.

Hearst (in press) has suggested that people and animals share a bias favoring the presence over the absence of a stimulus as a source of information (hereafter called an information-processing bias). In the case of people, that notion has considerable intuitive appeal as well as some empirical support (see Hearst, in press). The present view is that animals may also have such a bias, but the FPE is not a necessary consequence of it.

The notion that the FPE is due to an informationprocessing bias derives in part from superficial similarities between the FPE in human and animal learning experiments. In feature-learning experiments with human subjects, however, the response requirement usually serves only to inform us that the subject has or has not learned to distinguish between the positive and negative cases (see, e.g., Newman et al., 1980). Some animal discrimination problems may also require the subject to locate and identify the relevant discriminanda, but animals must also learn to respond differentially to $\mathrm{S}+$ and $\mathrm{S}-$. Thus, the response requirement serves to tell us both whether an animal can distinguish the positive from the negative stimulus and whether the subject can respond differentially on the basis of that information. A failure to respond correctly could indicate either a failure to learn the difference between the discriminanda or a failure to acquire the appropriate response tendencies. Here it is noteworthy that human subjects who, in a FN condition, do manage to discover the feature have no difficulty responding correctly to the two displays (Newman et al., 1980). In contrast, pigeons, the species for which we have the largest number of consistent demonstrations of the FPE, often discriminate between the positive and negative displays almost from the outset of training in both the FP and FN conditions (as evidenced by sign-tracking behavior); but, in the FN condition, they fail to withhold responding on $\mathbf{S}-$ trials. Thus, the FPE in pigeons, and perhaps in other animals as well, may often reflect a performance deficit in FN subjects rather than a deficit in the subjects' learning about the relationship between the feature and the availability of reinforcement (Hearst, in press).

An information-processing bias could explain why, in Phase 2 of Experiment 2 here, pretrial nonreinforcement, that is, the absence of reinforcement was almost completely ineffective as a discriminative stimulus. Note, however, that pretrial nonreinforcement was equally ineffective as a cue in both the FP and FN conditions. On the other hand, a pretrial reinforcement functioned quite well as a discriminative cue, with learning facilitated when that cue signaled an S- trial; that is, an FNE was obtained.

Whether one obtains a FPE or a FNE may depend on the relative value in a given task of a signal to respond versus one not to respond. This may be recognized as a restatement of the long-standing and as yet unresolved issue in animal discrimination learning concerning the relative behavioral control exercised by $\mathbf{S}+$ and $S_{-}$, which, as things stand, appears to vary from one discrimination problem to another. There are, however, several indications that $S-$ may exercise more control than $S+$ in runway or maze situations with rats. In Olton (1972), for example, avoidance of nonreinforcement was stronger than approach to reinforcement. And recent investigations of discrimination learning in the runway have reported that the very substantial effects of reward sequence and intertrial interval on behavior are due almost entirely to the effects of those vari- 
ables on responding to S- (e.g., Haggbloom, 1979, 1980, 1982; Haggbloom \& Tillman, 1980; Capaldi, Nawrocki, \& Verry, in press).

Mackintosh (1974) suggested that $S$ - might exercise relatively more control than $\mathrm{S}+$ to the extent that subjects cannot avoid responding to or contacting the S- cue, a characteristic of the experiments just cited and the two experiments reported here. In contrast, FN subjects in Jenkins and Sainsbury's (1969) experiments and in many subsequent featurelearning experiments could and did direct their behavior away from the feature on $\mathrm{S}$ - trials. Interestingly, Jenkins and Sainsbury (1969) noted that the FPE might be related to the relative control exercised by $\mathrm{S}+$ and $\mathrm{S}-$, but they found that thinking about the problem in that way was "more trouble than help in reaching some understanding of the results" (p. 240). Yet it may turn out that the FPE tends to occur in situations in which $\mathrm{S}+$ exercises a high degree of control, whereas an FNE is more likely to occur in situations in which $\mathrm{S}-$ exercises relatively greater control. Granted, the presence of a stimulus is likely to be generally more informative than its absence. It seems equally likely that there should be situations in which a signal that some other event, such as a reinforcement, was not available would be more valuable than a signal that the event was available. Whether we can better differentiate between these two cases in terms of the relative control exercised by $\mathrm{S}+$ and $\mathrm{S}-$, or in some other way, remains to be determined.

\section{REFERENCES}

Bottjer, S. W., \& Hearst, E. Food delivery as a conditional stimulus: Feature-learning and memory in pigeons. Journal of the Experimental Analysis of Behavior, 1979, 31, 189-207.

Capaldi, E. J., Nawrocki, T. M., \& Verry, D. R. Stimulus control in instrumental discrimination learning and reinforcement situations. Journal of Experimental Psychology: Animal Behavior Processes, in press.

Crowell, C. R., \& Bernhandt, T. P. The feature-positive effect and sign-tracking behavior during discrimination learning in the rat. Animal Learning \& Behavior, 1979, 7, 313-317.

Farthing, G. W. Discrimination of compound stimuli involving the presence or absence of a distinctive visual feature. Journal of the Experimental Analysis of Behavior, 1971, 16, 327-336.

HaGgBLoom, S. J. The differential reinforcement of rewardproduced and response-produced stimuli. Learning and Motivation, 1979, 10, 364-381.

Haggbloom, S. J. Resistance to discrimination and subsequent resistance to extinction as a function of the sequence of partial $\mathrm{S}+$ reward in differential conditioning. Animal Learning \& $\mathrm{Be}$ havior, 1980, 8, $441-446$.

HAGgBLOOM, S. J. Effect of N-R transitions during partial reinforcement pretraining on subsequent resistance to discrimination. Animal Learning \& Behavior, 1982, 10, 61-64.
Haggbloom, S. J., \& Tillman, D. J. Sequential effects on discrimination reversal. Learning and Motivation, 1980, 11, 318-338.

HalgRen, C. R. Latent inhibition in rats: Associative or nonassociative? Journal of Comparative and Physiological Psychology, 1974, 86, 74-78.

Hearst, E. Excitation, inhibition and discrimination learning. In N. J. Mackintosh \& W. K. Honig (Eds.), Fundamental issues in associative learning. Halifax: Dalhousie University Press, 1969.

HEARST, E. Stimulus relationships and feature selection in learning and behavior. In S. H. Hulse, H. Fowler, \& W. K. Honig (Eds.), Cognitive processes in animal behavior. Hillsdale, N.J: Erlbaum, 1978.

HeARST, E. Absence as information: Some implications for learning, performance, and representational processes. In $\mathbf{H}$. Roitblat, T. Bever, \& H. S. Terrace (Eds.), Animal cognition. Hillsdale, N.J: Erlbaum, in press.

Hearst, E., \& Jenkins, H. M. Sign-tracking: The stimulusreinforcer relation and directed action. Austin: The Psychonomic Society, 1974.

Hinson, R. E. Effects of UCS preexposure on excitatory and inhibitory rabbit eyelid conditioning: An associative effect of conditioned contextual stimuli. Journal of Experimental Psychology: Animal Behavior Processes, 1982, 8, 49-61.

Jenkins, H. M., \& Sainsbury, R. S. The development of stimulus control through differential reinforcement. In N. J. Mackintosh \& W. K. Honig (Eds.), Fundamental issues in associative learning. Halifax: Dalhousie University Press, 1969.

LEA, S. E. G. The non-occurrence of a stimulus as a signal. Quarterly Journal of Experimental Psychology, 1974, 26, 616-621.

MaCkINTOSH, N. J. The psychology of animal learning. New York: Academic Press, 1974.

Newman, J., WolfF, W. T., \& Hearst, E. The feature-positive effect in adult human subjects. Journal of Experimental Psychology: Human Learning and Memory, 1980, 6, 630-650.

Olton, D. S. Discrimination behavior in the rat: Differential effects of reinforcement and nonreinforcement. Journal of Comparative and Physiological Psychology, 1972, 79, 284-290.

PACE, G. M., \& McCoY, D. F. Effects of stimulus contact on the feature-positive effect. American Journal of Psychology, 1981, 94, 153-158.

ReBerg, D., \& LeClerc, R. A feature positive effect in conditioned suppression. Animal Learning \& Behavior, 1977, 5, 143-147.

Riccio, D. C., URda, M., \& Thomas, D. R. Stimulus control in pigeons based on proprioceptive stimuli from floor inclination. Science, 1966, 153, 434-436.

Sainsbury, R. S. Discrimination learning utilizing positive or negative cues. Canadian Journal of Psychology, 1973, 27, 46-57.

TeRRY, W. S., \& WAGNeR, A. R. Short-term memory for "surprising" vs. "expected" unconditioned stimuli in Pavlovian conditioning. Journal of Experimental Psychology: Animal Behavior Processes, 1975, 104, 122-133.

\section{NOTE}

1. Eighteen days of training were scheduled for Phase 2 of Experiment 1 , but a formaldehyde spill in the laboratory building after Day 17 prevented completion of the last day of training.

(Manuscript received February 15, 1983; revision accepted for publication May 11, 1983.) 